\title{
Erratum to: Existence of solutions of scalar field equations with fractional operator
}

\author{
Norihisa Ikoma
}

\section{Erratum to: J. Fixed Point Theory Appl. (2017) 19:649-690 DOI 10.1007/s11784-016-0369-x}

In the latter part of [2, Proposition 3.6 (i)], it is claimed that any weak solution of

$$
(1-\Delta)^{\alpha} u=f(u) \quad \text { in } \mathbf{R}^{N}, \quad u \in H^{\alpha}\left(\mathbf{R}^{N}\right)
$$

decays faster than any polynomial. However, the proof there is valid only for positive solutions since the function $g(x):=\left(f(u(x))-\left(1-\delta_{0}\right) u(x)\right)+$ may not be compactly supported.

We shall modify the proof and show the decay estimate of any weak solution of

$$
(1-\Delta)^{\alpha} u=f(x, u) \quad \text { in } \mathbf{R}^{N}, \quad u \in H^{\alpha}\left(\mathbf{R}^{N}\right) .
$$

Proposition 0.1. Let $f \in C\left(\mathbf{R}^{N} \times \mathbf{R}, \mathbf{R}\right)$ satisfy $|f(x, s)| \leq C\left(|s|+|s|^{2_{\alpha}^{*}-1}\right)$ for each $(x, s) \in \mathbf{R}^{N} \times \mathbf{R}$ and

$$
\limsup _{s \rightarrow 0} \sup _{x \in \mathbf{R}^{N}} \frac{f(x, s)}{s}<1 .
$$

Then for every weak solution $u$ of (1) and every $k \in \mathbf{N}$, there exists a $C_{k, u}$ such that $|u(x)| \leq C_{k, u}(1+|x|)^{-k}$ for all $x \in \mathbf{R}^{N}$.

We first introduce the extension problem (see [1]):

$$
\begin{cases}t^{1-2 \alpha}\left(-\Delta_{x}+1\right) w-\left(t^{1-2 \alpha} w_{t}\right)_{t}=0 & \text { in } \mathbf{R}_{+}^{N+1} \\ w=u & \text { on } \partial \mathbf{R}_{+}^{N+1}\end{cases}
$$

The online version of the original article can be found under doi:10.1007/ s11784-016-0369-x. 
where $\Delta_{x}=\sum_{i=1}^{N} \partial_{x_{i}}^{2}$. We also set

$$
\begin{aligned}
X^{\alpha} & :=\left\{w: \mathbf{R}_{+}^{N+1} \rightarrow \mathbf{R} \mid\|w\|_{X^{\alpha}}<\infty\right\}, \\
\|w\|_{X^{\alpha}}^{2} & :=\int_{\mathbf{R}_{+}^{N+1}} t^{1-2 \alpha}\left(|\nabla w|^{2}+w^{2}\right) \mathrm{d} X, \quad X:=(x, t)
\end{aligned}
$$

where $\nabla=\left(\nabla_{x}, \partial_{t}\right)$. Recall [2, Proposition 5.2]:

Proposition 0.2. (i) There exists the trace operator $\operatorname{Tr}: X^{\alpha} \rightarrow H^{\alpha}\left(\mathbf{R}^{N}\right)$.

(ii) For each $u \in H^{\alpha}\left(\mathbf{R}^{N}\right)$, there is a unique solution $E u \in X^{\alpha}$ of (3). In addition, there is a constant $\kappa_{\alpha}>0$ so that

$$
\kappa_{\alpha}\langle u, \operatorname{Tr} w\rangle_{\alpha}=\int_{\mathbf{R}_{+}^{N+1}} t^{1-2 \alpha}(\nabla(E u) \cdot \nabla w+(E u) w) \mathrm{d} X
$$

for every $u \in H^{\alpha}\left(\mathbf{R}^{N}\right)$ and $w \in X^{\alpha}$.

(iii) For each $u \in H^{\alpha}\left(\mathbf{R}^{N}\right)$ and $w \in X^{\alpha}$ with $\operatorname{Tr} w=u, \kappa_{\alpha}\|u\|_{\alpha}^{2}=$ $\|E u\|_{X^{\alpha}}^{2} \leq\|w\|_{X^{\alpha}}^{2}$ hold.

Now we prove Proposition 0.1:

Proof of Proposition 0.1. Let $u$ be a weak solution of (1). By (2), we may choose $s_{0}>0$ and $\delta_{0}>0$ such that

$$
\frac{f(x, s)}{s}<1-2 \delta_{0} \quad \text { for any }(x, s) \in \mathbf{R}^{N} \times\left(\left[-s_{0}, s_{0}\right] \backslash\{0\}\right) .
$$

From the former part of the proof of [2, Proposition 3.6 (i)], we see that $u \in C_{\mathrm{b}}^{\beta}\left(\mathbf{R}^{N}\right)$ for any $\beta \in(0,2 \alpha)$ and $u(x) \rightarrow 0$ as $|x| \rightarrow \infty$. Hence, we may choose an $R_{0}>0$ such that

$$
|x| \geq R_{0}, u(x) \neq 0 \quad \Rightarrow \quad \frac{f(x, u(x))}{u(x)} \leq 1-\delta_{0} .
$$

Denote by $\chi_{R_{0}}(x)$ the characteristic function of $B_{R_{0}}(0)$. Notice that $\chi_{R_{0}}(x)|f(x, u(x))| \in L^{2}\left(\mathbf{R}^{N}\right) \cap L^{\infty}\left(\mathbf{R}^{N}\right)$ is compactly supported. Let $v$ be a unique solution of

$$
(1-\Delta)^{\alpha} v-\left(1-\delta_{0}\right) v=\chi_{R_{0}}(x)|f(x, u(x))| \quad \text { in } \mathbf{R}^{N}, \quad v \in H^{\alpha}\left(\mathbf{R}^{N}\right) .
$$

Then [2, Proposition 5.1] asserts that for any $k \in \mathbf{N}$ there is a $c_{k}>0$ so that

$$
0<v(x) \leq c_{k}(1+|x|)^{-k} \quad \text { for all } x \in \mathbf{R}^{N} .
$$

Therefore, it suffices to prove $-v(x) \leq u(x) \leq v(x)$ for all $x \in \mathbf{R}^{N}$.

To this end, remark that $u$ satisfies

$$
(1-\Delta)^{\alpha} u=\chi_{R_{0}}(x) f(x, u(x))+\left(1-\chi_{R_{0}}(x)\right) f(x, u(x)) \text { in } \mathbf{R}^{N} .
$$

Setting $U(x):=v(x)-u(x)$, one finds that

$$
\begin{aligned}
(1-\Delta)^{\alpha} U= & \chi_{R_{0}}(x)\{|f(x, u(x))|-f(x, u(x))\} \\
& +\left\{-\left(1-\chi_{R_{0}}(x)\right) f(x, u(x))+\left(1-\delta_{0}\right) v(x)\right\} .
\end{aligned}
$$


Now consider $E U$ and $(E U)_{-}$where $w_{-}(X):=\max \{0,-w(X)\}$. It is easily seen that $(E U)_{-} \in X^{\alpha}$ and $\operatorname{Tr}(E U)_{-}=U_{-}$. Applying Proposition 0.2, we get

$$
\begin{aligned}
\kappa_{\alpha} & \left\langle U, U_{-}\right\rangle_{\alpha} \\
& =\int_{\mathbf{R}^{N}} t^{1-2 \alpha}\left(\nabla E U \cdot \nabla(E U)_{-}+(E U)(E U)_{-}\right) \mathrm{d} X=-\left\|(E U)_{-}\right\|_{X^{\alpha}}^{2} .
\end{aligned}
$$

On the other hand,

$$
\begin{aligned}
\kappa_{\alpha}\left\langle U, U_{-}\right\rangle_{\alpha}= & \kappa_{\alpha} \int_{\mathbf{R}^{N}}\left[\chi_{R_{0}}\{|f(x, u)|-f(x, u)\}\right. \\
& \left.+\left\{-\left(1-\chi_{R_{0}}\right) f(x, u)+\left(1-\delta_{0}\right) v\right\}\right] U_{-} \mathrm{d} x \\
\geq & \kappa_{\alpha} \int_{\mathbf{R}^{N}}\left(1-\chi_{R_{0}}\right)\left[-f(x, u)+\left(1-\delta_{0}\right) v\right] U_{-} \mathrm{d} x .
\end{aligned}
$$

If $U_{-}(x)>0$ and $|x| \geq R_{0}$, then from $u(x)>v(x)>0$ and (4) it follows that

$$
\begin{aligned}
& \left(1-\chi_{R_{0}}(x)\right)\left[-f(x, u(x))+\left(1-\delta_{0}\right) v(x)\right] \\
& \quad \geq\left(1-\chi_{R_{0}}(x)\right)\left[-\left(1-\delta_{0}\right) u(x)+\left(1-\delta_{0}\right) v(x)\right] \\
& \quad=\left(1-\delta_{0}\right)\left(1-\chi_{R_{0}}(x)\right) U(x)
\end{aligned}
$$

Hence,

$$
\kappa_{\alpha} \int_{\mathbf{R}^{N}}\left(1-\chi_{R_{0}}\right)\left[-f(x, u)+\left(1-\delta_{0}\right) v\right] U_{-} \mathrm{d} x \geq-\left(1-\delta_{0}\right) \kappa_{\alpha}\left\|U_{-}\right\|_{L^{2}}^{2} .
$$

Next, by the Plancherel theorem and Proposition 0.2, one sees

$$
\kappa_{\alpha}\left\|U_{-}\right\|_{L^{2}}^{2}=\kappa_{\alpha} \int_{\mathbf{R}^{N}}\left|\widehat{U_{-}}\right|^{2} \mathrm{~d} \xi \leq \kappa_{\alpha}\left\|U_{-}\right\|_{\alpha}^{2} \leq\left\|(E U)_{-}\right\|_{X^{\alpha}}^{2}
$$

Combining this with (4)-(6), we finally obtain

$$
-\left\|(E U)_{-}\right\|_{X^{\alpha}}^{2} \geq-\left(1-\delta_{0}\right)\left\|(E U)_{-}\right\|_{X^{\alpha}}^{2},
$$

which implies $(E U)_{-} \equiv 0$, hence, $U_{-} \equiv 0$ and $u(x) \leq v(x)$.

For the opposite inequality $-v(x) \leq u(x)$, we proceed similarly. Set $V(x):=v(x)+u(x)$. Then we have

$$
\begin{aligned}
-\left\|(E V)_{-}\right\|_{X^{\alpha}}^{2} & =\kappa_{\alpha}\left\langle V, V_{-}\right\rangle_{\alpha} \\
& \geq \kappa_{\alpha} \int_{\mathbf{R}^{N}}\left(1-\chi_{R_{0}}(x)\right)\left[f(x, u(x))+\left(1-\delta_{0}\right) v(x)\right] V_{-}(x) \mathrm{d} x .
\end{aligned}
$$

If $V_{-}(x)>0$ and $|x| \geq R_{0}$, then $u(x)<-v(x)<0$. Thus, by (4), one gets

$$
\begin{aligned}
& \left(1-\chi_{R_{0}}(x)\right)\left[f(x, u(x))+\left(1-\delta_{0}\right) v(x)\right] \\
& \quad \geq\left(1-\chi_{R_{0}}(x)\right)\left[\left(1-\delta_{0}\right) u(x)+\left(1-\delta_{0}\right) v(x)\right] \\
& \quad=\left(1-\delta_{0}\right)\left(1-\chi_{R_{0}}(x)\right) V(x) .
\end{aligned}
$$

The rest of the argument is same as the above and we obtain $V_{-} \equiv 0$. Thus, $-v(x) \leq u(x)$ holds and we complete the proof. 


\section{Acknowledgements}

This work was supported by JSPS KAKENHI Grant Number JP16K17623.

\section{References}

[1] Fall, M.M., Felli, V.: Unique continuation properties for relativistic Schrödinger operators with a singular potential. Discrete Contin. Dyn. Syst. 35(12), 5827$5867(2015)$

[2] Ikoma, N.: Existence of solutions of scalar field equations with fractional operator. J. Fixed Point Theory Appl. 19, 649-690 (2017)

\section{Norihisa Ikoma}

Faculty of Mathematics and Physics, Institute of Science and Engineering Kanazawa University

Kakuma

Kanazawa, Ishikawa 9201192

Japan

e-mail: ikoma@se.kanazawa-u.ac.jp 